\title{
Immobilized Metal Affinity Chromatography with Phosphopeptide Enrichment
}

National Cancer Institute

\section{Source}

National Cancer Institute. Immobilized Metal Affinity Chromatography with

Phosphopeptide Enrichment. NCI Thesaurus. Code C161793.

A method to purify phosphoproteins or phosphopeptides from a mixture that leverages their affinity to ferric ion-charged NTA-agarose beads. The mixture is loaded on the column at low pH and the phosphorylated proteins or peptides are eluted using alkaline buffers containing edetate at a pH range of 10-11 or phosphate-containing buffers. 